03

\title{
Влияние широкополосных флуктуаций скорости вращения на течения в сферических слоях
}

\author{
(С) Д.Ю. Жиленко, О.Э. Кривоносова \\ Институт механики МгУ им. М.В. Ломоносова, \\ 119192 Москва, Россия \\ e-mail: jilenko@imec.msu.ru
}

Поступило в Редакцию 14 декабря 2020 г.

В окончательной редакции 13 января 2021 r.

Принято к публикации 14 января 2021 г.

\begin{abstract}
Представлены результаты численного исследования изотермических осесимметричных течений вязкой несжимаемой жидкости во вращающихся сферических слоях при наличии случайных малоамплитудных колебаний скорости вращения внутренней сферы относительно постоянного во времени среднего значения. Рассмотрены два случая - вращение только внутренней сферы и однонаправленное вращение сфер с равными угловыми скоростями. Установлено, что в этих двух случаях течения по-разному реагируют на внесение возмущений. Проведено сравнение с экспериментом, подтверждающее обнаруженную в расчетах возможность увеличения средней скорости в отдельных областях течения с увеличением амплитуды флуктуаций.
\end{abstract}

Ключевые слова: шум, течения с вращением, сферическое течение Куэтта, управление неустойчивостью.

DOI: $10.21883 / J T F .2021 .06 .50862 .345-20$

\section{Введение}

Случайные флуктуации граничных условий всегда присутствуют в природных течениях и могут рассматриваться как внешний по отношению к этим течениям шум. Именно влиянием шума принято объяснять, например, изменение режимов атмосферной циркуляции, разогрев стратосферы и увеличение образования озона в атмосфере [1 4]. Известно, что внешний шум способствует обнаружению слабых сигналов, их усилению и параметрической генерации колебаний [5-8]. Также внешний шум может использоваться в технологиях извлечения энергии из низкоскоростных ветровых и волновых потоков [9]. Присутствие шума является неотъемлемой частью некоторых моделей природных процессов, таких, например, как генерация магнитного поля Солнца под действием произвольных неосесимметричных возмущений в сферическом течении идеально проводящей среды [10]. Добавление шума в течение вязкой несжимаемой жидкости увеличивает порог генерации магнитного поля [11], во вращающихся сферических слоях приводит при определенных условиях к смене волнового числа после потери устойчивости течения [12]. Несмотря на определенные успехи прямого численного моделирования воздействия шума на течения [11,13], открытым остается вопрос о способах определения устойчивости зашумленных течений, в том числе в рамках линеаризованных уравнений Навье-Стокса [14]. Известно, что периодические колебания скорости вращения приводят к изменению профилей азимутальной скорости, что достаточно хорошо изучено для рассматриваемых в настоящей работе течений вязкой несжимаемой жидкости во вращающихся сферических слоях [15-19], однако для случайных флуктуаций скорости вращения таких результатов нет. Цель настоящей работы состоит в численном исследовании влияния случайных флуктуаций со средним во времени нулевым значением на изменение свойств осесимметричных течений вязкой несжимаемой жидкости во вращающихся сферических слоях на основе решения как полной системы уравнений Навье-Стокса, так и анализа линейной устойчивости по отношению к трехмерным возмущениям в виде бегущих азимутальных волн для определения направления и величины смещения предела устойчивости под влиянием шума.

\section{1. Метод численного исследования}

Изотермические течения вязкой несжимаемой жидкости во вращающемся сферическом слое описываются уравнениями Навье-Стокса и неразрывности:

$$
\frac{\partial \mathbf{U}}{\partial t}=\mathbf{U} \operatorname{rot} \mathbf{U}-\operatorname{grad}\left(\frac{p}{\rho}+\frac{\mathbf{U}^{2}}{2}\right)-v \operatorname{rotrot} \mathbf{U}, \quad \operatorname{div} \mathbf{U}=0 .
$$

Здесь $\mathbf{U}$ - поле скорости, $p$ - давление, оба параметра являются функциями времени и координат, $\rho-$ плотность, $v$ - кинематическая вязкость жидкости в слое. Граничные условия прилипания и непротекания в сферической системе координат с радиальным $(r)$, полярным $(\theta)$ и азимутальным $(\varphi)$ направлениями имеют вид

$$
\begin{gathered}
u_{\varphi}\left(r=r_{k}\right)=\Omega_{k}(t) r_{k} \sin \theta \\
u_{r}\left(r=r_{k}\right)=0, \quad u_{\theta}\left(r=r_{k}\right)=0, \quad k=1,2,
\end{gathered}
$$

где $u_{\varphi}, u_{r}, u_{\theta}$ - соответственно азимутальная, радиальная и полярная компонента скорости, $r_{k}, \Omega_{k}(k=1,2)-$ 
радиусы и угловые скорости вращения сфер: $k=1$ внутренней и $k=2$ внешней. Численный метод решения основан на консервативной конечно-разностной схеме дискретизации уравнений Навье-Стокса по пространству и полунеявной схеме Рунге-Кутты 3-го порядка точности для интегрирования по времени [20]. Решалась система уравнений, описывающая нестационарные течения, симметричные относительно плоскости экватора и оси вращения. При расчете использовались неравномерные по $r$ сетки, с общим количеством узлов $(1.4-1.8) \cdot 10^{4}$, чувствительность рассчитываемых величин к размерам сетки подробно рассматривалась в [21]. Во всех рассматриваемых случаях дополнительный шум вносился в течение в виде случайных флуктуаций скорости вращения внутренней сферы: $\Omega_{1}(t)=\Omega_{10}(1+\operatorname{Nrn}(j))$. Здесь $\Omega_{10}$ - постоянная во времени осредненная часть скорости вращения внутренней сферы, $N$ - амплитуда шума и $r n(j)$ - псевдослучайное число из последовательности длиной $M$, изменяемое на каждом шаге по времени. Так же как и в [13], одна и та же последовательность $M$ случайных чисел со стандартным нормальным распределением использовалась во всех расчетах. Линейная устойчивость осесимметричных течений изучалась по отношению к трехмерным возмущениям в виде бегущих азимутальных волн с азимутальными числами $m=3,4$. Система линеаризованных относительно возмущений уравнений интегрировалась по времени до выхода решения на асимптотическое поведение $\propto \exp (\sigma t)$ [21]. Действительная часть $\sigma_{r e}-$ инкремент нарастания/затухания возмущения, мнимая часть определяет фазовую угловую скорость рассматриваемой моды $C_{\omega}=-\sigma_{i m} / m$. Расчеты проведены при параметpax, соответствующих условиям проведения экспериментов [12]: $v=5 \cdot 10^{-5} \mathrm{~m}^{2} / \mathrm{s}, r_{1}=0.075 \mathrm{~m}, r_{2}=0.15 \mathrm{~m}$ (толщина сферического слоя равна радиусу внутренней сферы). Продолжительность одного варианта расчета составляла $2.4 \cdot 10^{3} \mathrm{~s}$ с момента начала внесения шума в сигнал стационарного вращения внутренней сферы. Результаты расчетов представлены ниже в зависимости от безразмерных параметров, используемых ранее в [12]: чисел Рэйнольдса $\operatorname{Re}_{k}=\Omega_{k 0} r_{k}^{2} / \nu$, определяемых для внутренней $(k=1)$ и внешней $(k=2)$ сфер, и амплитуды шума

$$
N=\frac{1}{\Omega_{10}} \sqrt{\frac{1}{K-1} \sum_{i=1}^{K}\left(\Omega_{1}\left(t_{i}\right)-\Omega_{10}\right)^{2}} .
$$

Здесь $K$ - длина временной выборки.

\section{2. Методика проведения эксперимента}

Сферический слой в эксперименте образован зазором между внутренней и внешней сферическими оболочками. Сферы и рабочая жидкость, заполняющая зазор между ними, оптически прозрачны. Рабочая жидкость - силиконовое масло, в которое в качестве светорассеивающей примеси для визуализации структуры течения добавлено немного (менее $0.002 \%$ по объему) алюминиевой пудры. Температура рабочей жидкости измеряется датчиком, установленным в экваториальной плоскости внешней сферы. Для ограничения колебаний температуры рабочей жидкости выше $\pm 0.05^{\circ} \mathrm{C}$ сферы размещены в термостате, через который прокачивается подогреваемое или охлаждаемое силиконовое масло. Вращение сфер регулируется цифровой системой управления с выделенным сигнальным процессором, которая обеспечивает как сохранение среднего значения угловой скорости с точностью $\pm 0.05 \%$, так и величину отклонения от среднего значения. Мгновенные значения угловой скорости каждой из сфер $\Omega_{k}(t)$ вычисляются путем дифференцирования по времени сигналов фазы, поступающих с расположенных на валах датчиков скорости вращения. В случае если в системе управления задается ненулевая амплитуда шума, величина $\Omega_{1}(t)$ начинает изменяться во времени по отношению к заданному значению. Это изменение происходит путем добавления нормированной величины (которая может быть как положительной, так и отрицательной), выдаваемой генератором случайных чисел. При этом среднее значение $\Omega_{10}$ остается неизменным во времени. Величина контролируемой системой управления амплитуды шума $N$, вносимого в течение случайными флуктуациями скорости вращения внутренней сферы, изменяется в диапазоне 0.0095-0.06. Как в отсутствие, так и в присутствии дополнительно вносимого шума, спектр величины $\Omega_{1}(t)$ имеет равномерно распределенную амплитуду в широком диапазоне частот по обе стороны от малоамплитудного пика на частоте $4.5 \mathrm{~Hz}$ (рис. 2 из работы [12]). Азимутальная компонента скорости течения измерялась цифровым лазерным доплеровским анемометром, выполненным с использованием оптической схемы прямого рассеивания. Измерения проводились в точке течения, расположенной на расстоянии $0.078 \mathrm{~m}$ от экваториальной плоскости и $0.105 \mathrm{~m}$ от оси вращения. Сигнал скорости течения записывался на ПК с частотой опроса $25.45 \mathrm{~Hz}$ с целью последующей обработки. Более подробное описание деталей эксперимента представлено в [12].

\section{3. Результаты}

В настоящей работе рассматривается воздействие шума с малой амплитудой $(N<0.18$ в расчетах и $N<0.06$ в эксперименте). В каждый момент времени скорость вращения - случайная величина, но в соответствии с указанными выше условиями прилипания на границах, передаваемые в течение возмущения, не зависят от азимутального угла и симметричны относительно плоскости экватора. Поэтому рассматриваемые нестационарные течения, так же как и в отсутствие шума, до потери устойчивости остаются симметричными относительно 


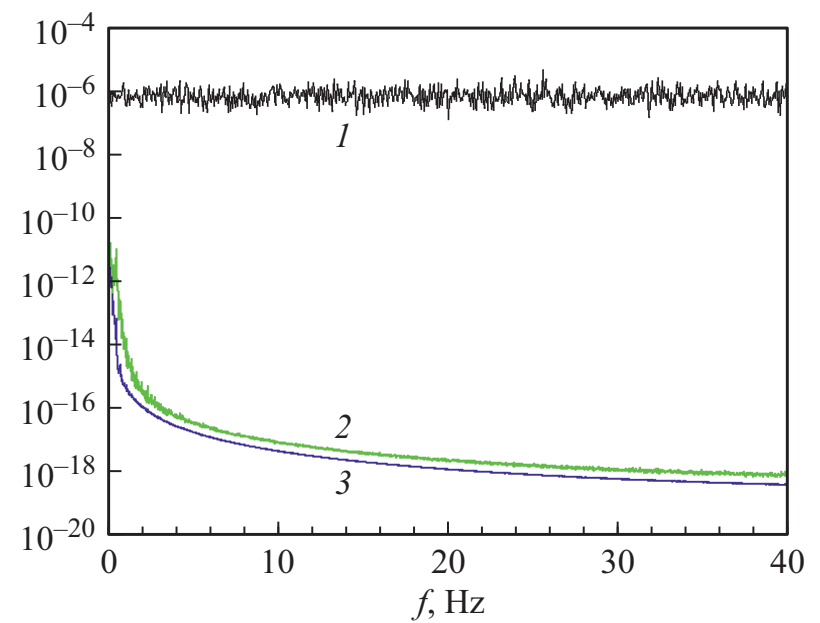

Pис. 1. Спектры: $1-$ флуктуаций скорости вращения в первом и втором случае, 2,3 - флуктуаций азимутальной компоненты скорости вращения в точке слоя, удаленной от внутренней сферы на расстояние $0.1253 \mathrm{~m}, \theta=45.75^{\circ} .2-\mathrm{Re}_{1}=250$, $\mathrm{Re}_{2}=1000, N=0.04 ; 3-\mathrm{Re}_{1}=350, \mathrm{Re}_{2}=0, N=0.04$.

экватора и оси вращения [22]. Рассматривается воздействие шума на два класса течений. В первом случае течение формируется при вращении только внутренней сферы $\Omega_{10}=3.1-4.35 \mathrm{~s}^{-1}$, внешняя неподвижна: $\mathrm{Re}_{2}=0$. Во втором случае течение формируется при однонаправленном вращении сфер с равными угловыми скоростями: $\operatorname{Re}_{2}=4 \operatorname{Re}_{1}\left(\Omega_{10}=\Omega_{20}=0.71-8.89 \mathrm{~s}^{-1}\right)$. Кинетическая энергия течения в сферическом слое $E$ может быть представлена в виде суммы двух составляющих: азимутальной $E_{\varphi}$ и меридиональной $E_{\psi}$, которые определяются интегрированием по всему объему сферического слоя соответствующих компонент скорости течения:

$$
E_{\varphi}=\int u_{\varphi}^{2}, \quad E_{\psi}=\int\left(u_{r}^{2}+u_{\theta}^{2}\right) .
$$

Рассматриваемые два класса течений существенно отличаются друг от друга соотношением $E_{\varphi} / E_{\psi}$. При $\mathrm{Re}_{2}=0$ существенны все три компоненты скорости и $E_{\varphi} / E_{\psi} \approx 10$, при $\operatorname{Re}_{2}=4 \operatorname{Re}_{1}$ течение близко к „твердотельному“ вращению, т. е. радиальная и меридиональная компоненты очень малы, и $E_{\varphi} / E_{\psi} \approx 10^{10}$. В отсутствие шума в первом случае течение теряет устойчивость при сравнительно небольшой величине $\mathrm{Re}_{1}$ с формированием бегущих азимутальных волн [22], во втором случае течение теряет устойчивость при очень больших числах Рейнольдса [23], и в настоящей работе его неустойчивость не рассматривается. В случае вращения только внутренней сферы экспериментально показано [12], что внесение шума не изменяет вида вторичного течения, и на пределе устойчивости в рассматриваемом слое с зазором, равным радиусу внутренней сферы, формируются бегущие азимутальные волны с зависящими от начальных условий волновыми числами $m=3$ или 4 .

В обоих рассматриваемых случаях спектр флуктуаций $\Omega_{1}(t)$ представляет собой „белый шум“, т. е. имеет равную амплитуду в широком диапазоне частот (рис. 1). Вид случайных флуктуаций скорости течения изменяется по мере удаления от внутренней сферы, и амплитуды спектров скорости в обоих случаях экспоненциально затухают с увеличением частоты (рис. 1). Отличия этих спектров по сравнению с подводимым „белым“ шумом объясняются тем, что так же как и в случае периодических колебаний внутренней сферы относительно состояния покоя [24], распространяющиеся в радиальном направлении высокочастотные возмущения скорости течения затухают быстрее, чем низкочастотные. В случае периодических колебаний скорости вращения внутренней сферы как в ламинарных течениях до потери устойчивости [19], так и в турбулентных течениях [18] добавление шума может приводить к изменению профилей средней скорости, а также к возрастанию скорости в локальных областях течения. Рассмотрим восприимчивость различных компонент скорости течений к шуму. На рис. 2 показана полученная в расчетах и экспериментах зависимость относительного приращения средней азимутальной скорости $V_{\varphi}$ от уровня шума в точке течения на средних широтах вблизи внешней сферы. Здесь $V_{\varphi}=\left(u_{\varphi n}-u_{\varphi 0}\right) / u_{\varphi 0}, u_{\varphi n}-$ азимутальная скорость течения в присутствии шума, $u_{\varphi 0}$ - та же величина без шума (в случае эксперимента при минимально возможном уровне шума). Видно, что при добавлении шума в случае $\operatorname{Re}_{2}=0$ (рис. 2 , кривые 1,2$)-V_{\varphi}$ увеличивается быстрее по сравнению со случаем $\operatorname{Re}_{2}=4 \mathrm{Re}_{1}$. Если же мы рассмотрим влияние шума на относительное изменение компонент скорости, лежащих в меридиональной плоскости течения $V_{m e r}=\left(u_{m}-u_{m 0}\right) / u_{m 0}$, где $u_{m}=\left(u_{r}^{2}+u_{\theta}^{2}\right)^{1 / 2}$, то ситуация меняется: при $\operatorname{Re}_{2}=4 \operatorname{Re}_{1}$ величина $V_{m e r}$ на 6 порядков больше и возрастает быстрее, чем в случае $\operatorname{Re}_{2}=0$. Таким образом, на средних широтах вблизи внешней сферы в первом из рассматриваемых случаев $\left(\operatorname{Re}_{2}=0\right)$ под воздействием

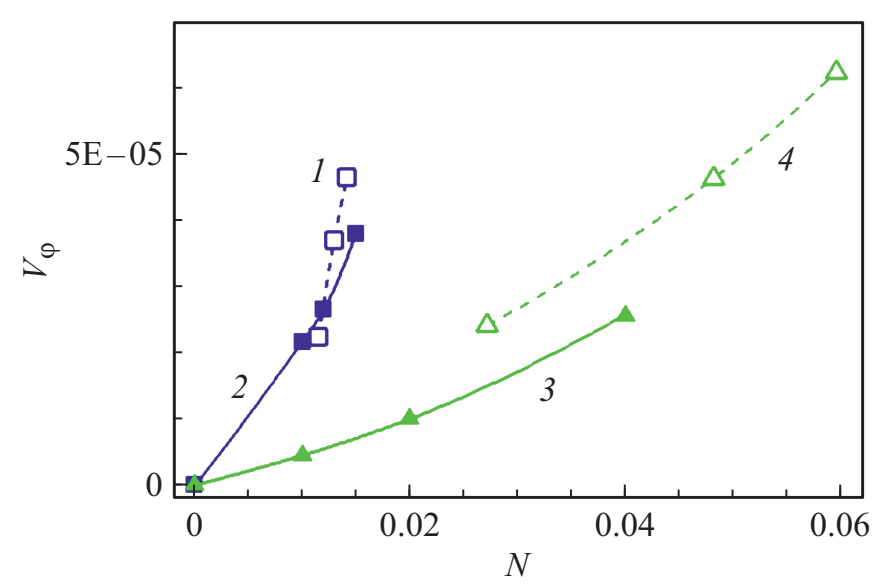

Рис. 2. Относительное приращение азимутальной компоненты скорости течения $V_{\varphi}$ под действием шума $N$ в точке течения, расположенной в соответствии с рис. 1: $1,2-\mathrm{Re}_{1}=450$, $\operatorname{Re}_{2}=0 ; 3,4-\operatorname{Re}_{1}=112.5, \operatorname{Re}_{2}=450.2,3-$ расчет, 1,4эксперимент. 

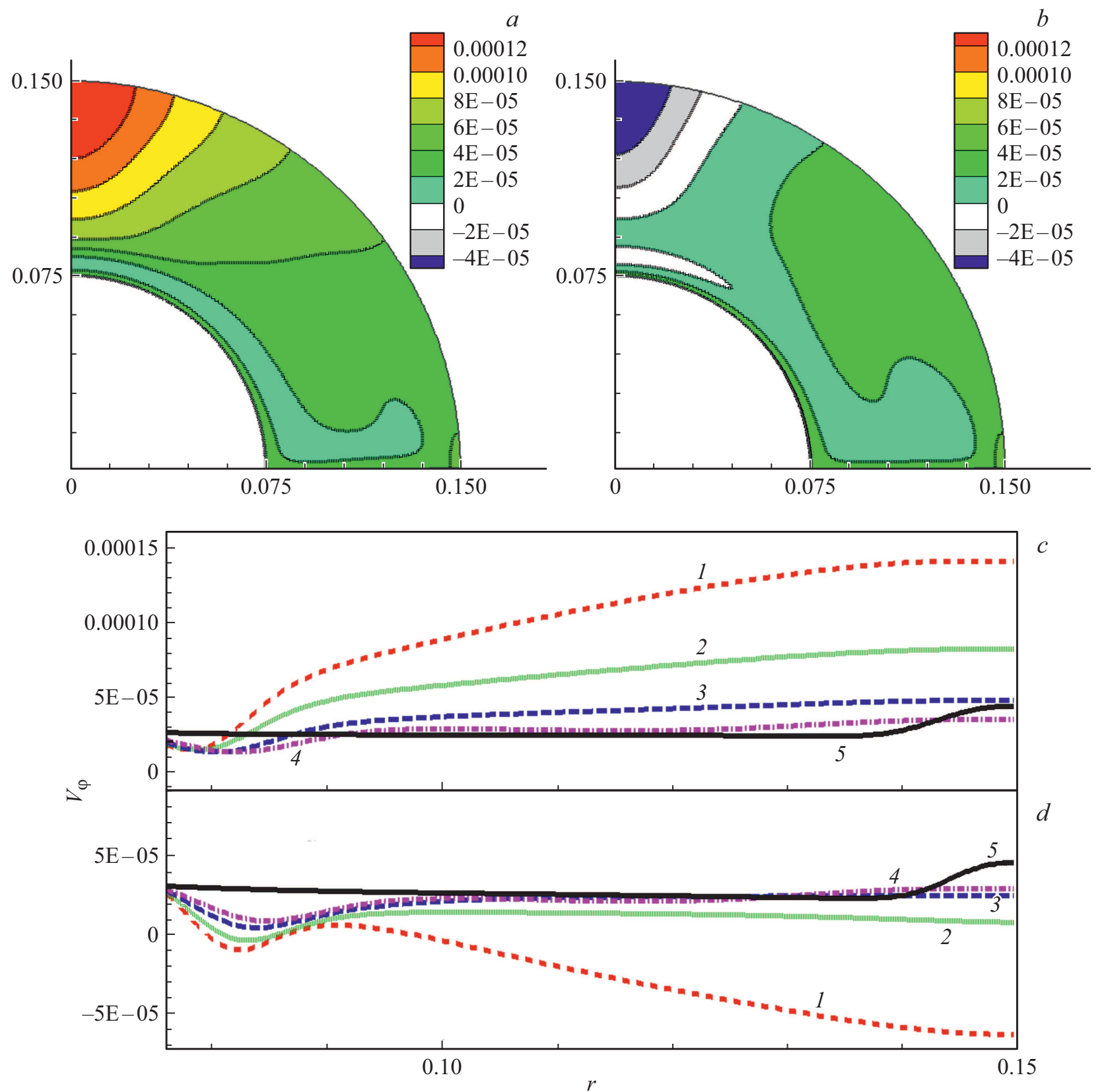

Рис. 3. $V_{\varphi}$ при $\operatorname{Re}_{1}=400, \operatorname{Re}_{2}=0(a, c)$ и $\operatorname{Re}_{1}=484, \operatorname{Re}_{2}=0(b, d)$ в меридиональной плоскости $(a, b)$ и в зависимости от расстояния между сферическими границами $(c, d)$ при различных отклонениях от оси вращения по меридиональному углу $\varphi: 1-$ $3,2-22.5,3-45,4-67.5,5-90^{\circ}$.

шума быстрее увеличивается $V_{\varphi}$, тогда как во втором $\left(\operatorname{Re}_{2}=4 \operatorname{Re}_{1}\right)-V_{\text {mer. }}$. Таким образом, восприимчивость различных компонент скорости к шуму зависит от конкретного течения.

Изменение скорости неоднородно по пространству и зависит не только от амплитуды шума $N$, но и от чисел Рэйнольдса. Так, в случае $\mathrm{Re}_{2}=0$ наиболее сильное влияние шума на изменение скорости $V_{\varphi}$ наблюдается в области течения вблизи оси вращения и внешней сферы. На рис. 3 представлена величина $V_{\varphi}$ при одном и том же уровне шума, но разных числах $\mathrm{Re}_{1}$. При меньшем $\mathrm{Re}_{1}$ (рис. $3, a, c) V_{\varphi}>0$ во всем зазоре, вблизи оси вращения и внешней сферы наблюдается наибольший прирост, наименьший - вблизи внутренней сферы и плоскости экватора, вследствие чего на зависимости $V_{\varphi}$ от радиуса появляются локальные минимумы (рис. 3,c). Под влиянием шума компоненты скорости могут не только увеличиваться, но и уменьшаться. Так, при повышении числа $\mathrm{Re}_{1}$ до величин, соответствующих, как далее будет показано, пределу устойчивости, наибольшее снижение величины $V_{\varphi}$ (рис. $3, b, d$ ) наблюдается в приполярной области, тогда как небольшое увеличение - вблизи плоскости экватора. Отметим, что наблюдаемое влияние шума на изменение профилей азимутальной скорости 


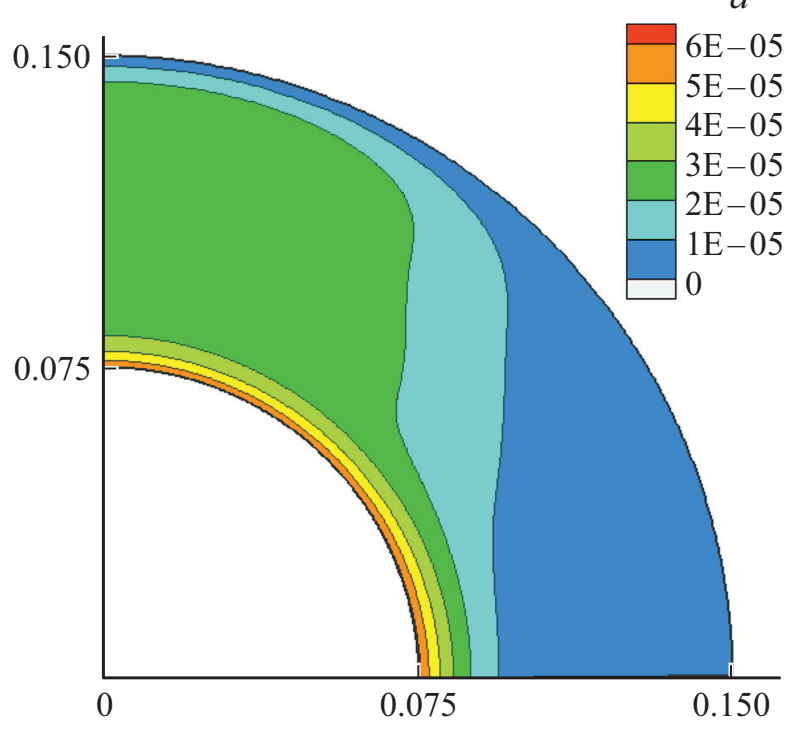

$b$

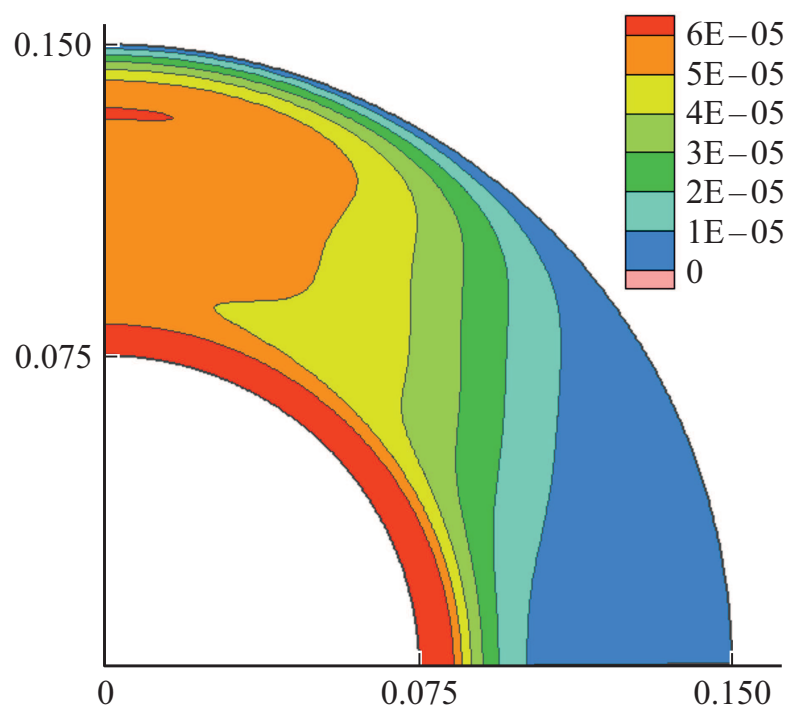

Рис. 4. $V_{\varphi}$ в меридиональной плоскости при $\operatorname{Re}_{1}=112.5, \operatorname{Re}_{2}=450 ; a-N=0.02, b-N=0.04$.

качественно соответствует влиянию периодических колебаний скорости вращения на изменение средней скорости в турбулентном течении [18], где наибольший прирост азимутальной компоненты наблюдался на экваторе вблизи внутренней сферы и мог уменьшаться по мере удаления от плоскости экватора. Уменьшение скорости возможно и во втором случае $\left(\operatorname{Re}_{2}=4 \mathrm{Re}_{1}\right)$. Так, во всем исследуемом диапазоне изменений $\operatorname{Re}_{1}$ и $N$ в середине слоя на низких широтах наблюдается небольшая область течения, в которой $V_{m e r}<0$. Наоборот, величина $V_{\varphi}$ во втором случае всегда положительна, влияние шума на ее изменение сосредоточено в узком слое вблизи внутренней сферы и приполярной области внешней сферы (рис. 4), практически не распространяясь за пределы цилиндрического стюартсоновского слоя [22], в котором происходит сглаживание сдвига азимутальной компоненты скорости.

Отмеченные выше особенности влияния шума на изменение скорости течения приводят к необходимости учета влияния шума на кинетическую энергию течений. Так, во втором случае $\left(\operatorname{Re}_{2}=4 \mathrm{Re}_{1}\right)$ азимутальная компонента кинетической энергии течения слабо изменяется с возрастанием амплитуды шума (рис. 5, $a$, отличия между $N=0$ и 0.04 существенно меньше величины символов) и зависит от числа Рэйнольдса в виде $E_{\varphi} \sim\left(\operatorname{Re}_{1}\right)^{2}$. В то же время $E_{\varphi}$ при максимальной амплитуде шума возрастает на 2 порядка по сравнению со случаем $N=0$ (рис. $5, b)$. И в первом, и во втором случаях при одном и том же уровне шума изменение кинетической энергии практически не зависит от чисел Рэйнольдса. Несмотря на то, что флуктуации скорости вращения передаются в течение через возмущения азимутальной компоненты течения, в обоих рассматриваемых случаях возрастание $N$ приводит к уменьшению соотношения $E_{\varphi} / E_{\psi}$. Но если в первом случае величина

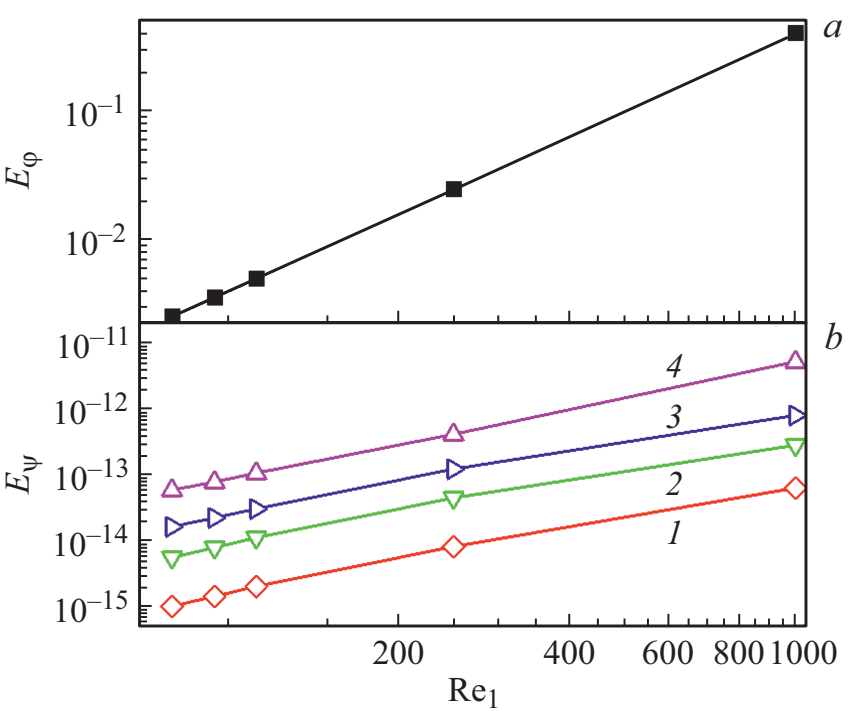

Рис. 5. Компоненты азимутальной $(a)$ и меридиональной $(b)$ кинетической энергии течения во втором случае $\left(\mathrm{Re}_{2}=4 \mathrm{Re}_{1}\right)$ в зависимости от $\mathrm{Re}_{1}: 1-N=0,2-N=0.01$, $3-N=0.02,4-N=0.04$.

уменьшения порядка $10^{-4}$ (рис. $6, a$ ), то во втором более $10^{1}$ (рис. $6, b$ ).

Индуцированные влиянием шума изменения полей средней скорости течения, его кинетической энергии и соотношения между $E_{\varphi}$ и $E_{\psi}$ могут приводить к смещению предела устойчивости. Даже в наиболее изученном экспериментально и численно случае вращения только внутренней сферы имеющиеся к настоящему времени экспериментальные данные не позволяют сделать выводы ни о направлении, ни о величине смещения предела устойчивости под влиянием искусственно вносимого в 


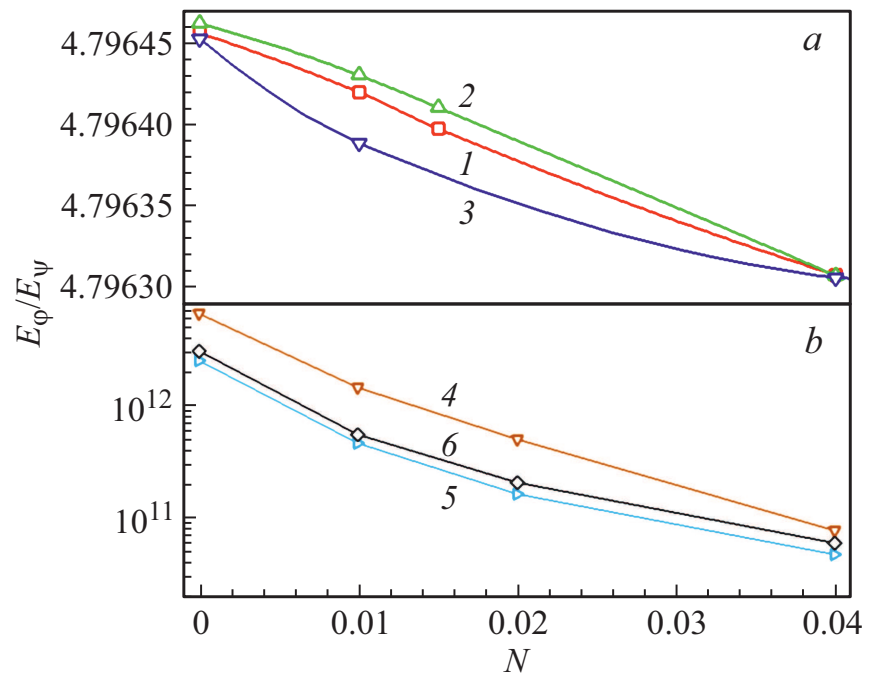

Pис. 6. Отношение азимутальной компоненты кинетической энергии течения к меридиональной в зависимости от амплитуды шума: $a-\operatorname{Re}_{2}=0: 1-\operatorname{Re}_{1}=350,2-400,3-484$; $b-\operatorname{Re}_{2}=\operatorname{Re}_{1}: 4-112.5,5-250,6-1000$.

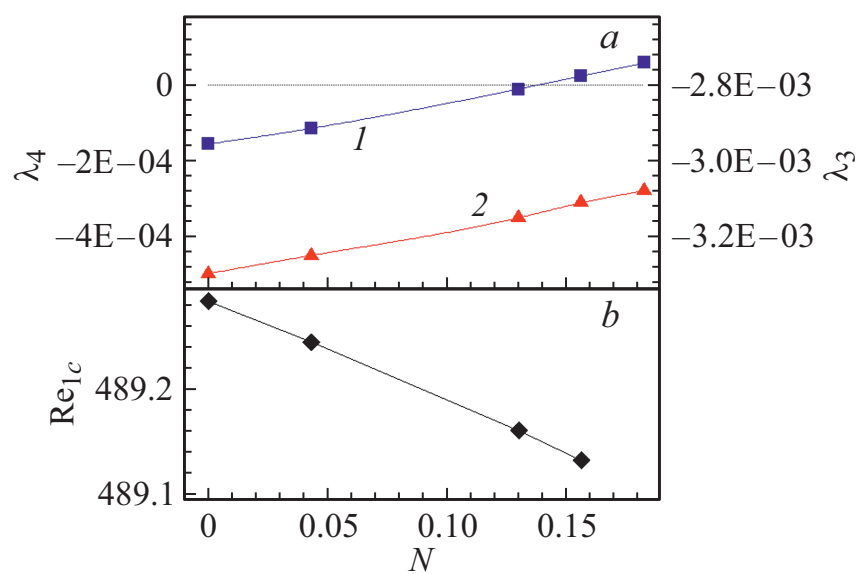

Pис. 7. $a-$ зависимости инкрементов нарастания/затухания $\lambda$ от амплитуды шума $N$ при $\operatorname{Re}_{1}=489.15$ для волновых чисел $m=4$ (1, левая ось) и $m=3(2$, правая ось); $b-$ зависимость критических значений числа Рейнольдса от амплитуды шума.

течение шума. Так, ни в экспериментах [12], где шум вносился флуктуациями скорости вращения, ни в более ранней работе [25], где периодические колебания с частотами в диапазоне $1-7 \mathrm{~Hz}$ вносились в течение акустическим динамиком, сдвиг предела устойчивости под влиянием флуктуаций не обнаружен. Это может быть вызвано тем, что положение предела устойчивости в $[12,19,25]$ определялось из соотношения $A \propto\left(\operatorname{Re}-\operatorname{Re}_{c}\right)^{1 / 2}[26]$, где $A$ - амплитуда периодических колебаний на собственной частоте линейной моды, a $\mathrm{Re}-\mathrm{Re}_{c}-$ величина надкритичности. В свою очередь, указанное выше соотношение в присутствии шума, как предполагается в [27], может изменяться. В таком случае в эксперименте необходимо измерять малые величины $A$ при $\left(\mathrm{Re}-\mathrm{Re}_{c}\right) \rightarrow 0$, что, как отмечалось в [19], затруднительно вследствие увеличения времени переходных процессов. Поэтому положение предела устойчивости в настоящей работе определяется численно, в соответствии с линейной теорией. Рассматривается, как наиболее изученное, в том числе с добавлением шума, течение при $\operatorname{Re}_{2}=0$. Даже при более простых, чем шум, периодических граничных условиях строгая постановка задач линейной устойчивости возможна только в некоторых частных случаях: это либо двумерные течения, либо трехмерные, но в прямолинейных каналах с параллельными стенками [28]. Поэтому при определении предела устойчивости течений с зависящими от времени граничными условиями используются различные приближенные подходы. Так, в [21] при низкочастотных периодических колебаниях скорости вращения внешней сферы анализ линейной устойчивости проводился в квазистационарном приближении, при котором предполагается, что величины инкрементов возрастания/затухания определяются полем скорости в текущий момент времени. В настоящей работе ситуация другая, поскольку присутствуют случайные колебания и на низких, и на высоких частотах. В связи с этим мы используем рассмотренный в [8] подход, при котором вместо системы с флуктуациями рассматривается система с осредненными параметрами, формируемая под влиянием этих флуктуаций. При таком подходе задача линейной устойчивости решается для осредненного во времени осесимметричного течения. Результаты показывают, что при одних и тех же $\mathrm{Re}_{1}$ величины инкрементов нарастания/затухания $\lambda$ монотонно растут с увеличением амплитуды шума (рис. 7,a), что качественно соответствует экспериментальным результатам [12]. При этом, также как и в отсутствие шума, доминирующим остается волновое число $m=4$ : при одних и тех же числах $\mathrm{Re}_{1}$ и уровне шума $\mathrm{N}$ инкременты нарастания для моды с $m=4$ выше, чем аналогичные величины для моды $m=3$ (рис. 7,a). При постоянных значениях шума $N$ рост кинетической энергии течения сопровождается одновременным возрастанием инкрементов $\lambda$ (рис. 8 ), $\lambda<0$ соответствует устойчивым

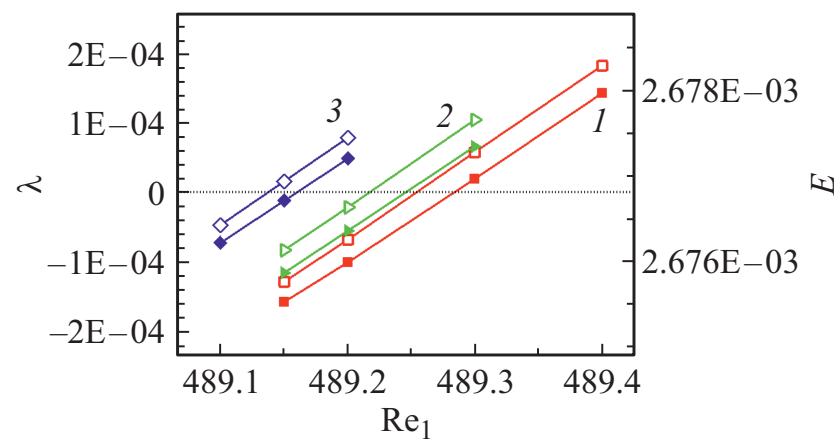

Рис. 8. Зависимость инкрементов нарастания $\lambda$ (темные символы, левая ось) и кинетической энергии течения $E$ (светлые символы, правая ось) от числа $\mathrm{Re}_{1} .1-N=0,2-$ $N=0.043,3-N=0.13$. Горизонтальная прерывистая линия соответствует $\lambda=0$. 
течениям, а $\lambda>0$ неустойчивым, условие $\lambda=0$ соответствует критическим значениям числа Рэйнольдса $\operatorname{Re}_{c}$. Как видно на рис. $8, c$ увеличением $N$ условие $\lambda=0$ начинает выполняться при меньших величинах $\operatorname{Re}_{1}$. Это означает, что с увеличением шума положение предела устойчивости (рис. $7, b)$ смещается в область меньших чисел $\operatorname{Re}_{1}$.

\section{Заключение}

Результаты проведенных численных исследований свидетельствуют о том, что широкополосные случайные флуктуации скорости вращения вызывают увеличение кинетической энергии течения. Наблюдается увеличение скорости течения в отдельных локальных областях, что подтверждается результатами экспериментов. Однако в случае вращения только внутренней сферы по мере приближения числа Рейнольдса к пределу устойчивости может наблюдаться локальное уменьшение скорости течения по сравнению со случаем отсутствия шума. Установлено, что добавление шума может приводить к повышению „трехмерности“ течения. Так, при равных угловых скоростях вращения сфер с увеличением амплитуды шума в так называемом „твердотельном“ вращении наблюдается многократное возрастание изначально очень малой по величине меридиональной компоненты кинетической энергии течения. Наоборот, при одном и том же уровне шума азимутальная компонента увеличивается на порядок меньше, чем в случае вращения только внутренней сферы с интенсивной меридиональной циркуляцией.

Проведенный для этого случая анализ линейной устойчивости осредненных во времени осесимметричных течений по отношению к трехмерным возмущениям показал, что величины инкрементов нарастания/затухания линейных мод возрастают с увеличением кинетической энергии течения и соответственно достигают положительных значений при меньших, чем в отсутствие шума, числах Рейнольдса. Это означает возможность уменьшения предела устойчивости течения при добавлении случайных широкополосных флуктуаций к постоянной во времени средней скорости вращения.

\section{Финансирование работы}

Работа выполнена при частичной финансовой поддержке Российского фонда фундаментальных исследований, проекты № 18-08-00074 и № 19-05-00028.

\section{Конфликт интересов}

Авторы заявляют, что у них нет конфликта интересов.

\section{Список литературы}

[1] P. Sura, M. Newman, C. Penland, P.J. Sardeshmukh. Atmosph. Sci., 62, 1391 (2005). DOI: 10.1175/JAS3408.1

[2] V. Lucarini, T. Bodai. Nonlinearity, R32 (2017). DOI: $10.1088 / 1361-6544 / a a 6 b 11$

[3] T. Birner, P.D. Williams. J. Atmosph. Sci., 65, 3337 (2008). DOI: $10.1175 / 2008 J A S 2770.1$

[4] Z. Liu, Y-C. Lai, J.M. Lopez. Chaos, 12 (2), 417 (2002). DOI: $10.1063 / 1.1476948$

[5] S.M. Bezrukov, I. Vodyanoy. Nature, 378, 362 (1995). DOI: $10.1038 / 378362$ ao

[6] О.В. Геращенко, С.Л. Гинзбург, М.А. Пустовойт. Письма в ЖЭТФ, $67(11), 945$ (1998). [O.V. Gerashenko, S.L. Ginzburg, M.A. Pustovoit. JETP Lett., 67 (11), 997 (1998).] DOI: $10.1134 / 1.567779$

[7] В.Н. Скоков, В.П. Коверда. ЖТФ, 84 (5), 9 (2014). [V.N. Skokov, V.P. Koverda. Tech. Phys., 59(5), 637 (2014).] DOI: $10.1134 / \mathrm{S} 1063784214050296$

[8] П.С. Ланда, А.А. Заикин. ЖЭТФ, 111 (1), 358 (1997). [P.S. Landa, A.A. Zaikin. JETP, 84 (1), 197 (1997).] DOI: $10.1134 / 1.558137$

[9] T. Morita, T. Omori, Y. Nakayama, S. Toyabe, T. Ishikawa. Phys. Rev. E, 101, 063101 (2020). DOI: 10.1103/PhysRevE.101.063101

[10] V.V. Pipin, A.G. Kosovichev. Ap. J., 867, 145 (2018). DOI: $10.3847 / 1538-4357 / \mathrm{aae} 1 \mathrm{fb}$

[11] J.-P. Laval, P. Blaineau, N. Leprovost, B. Dubrulle, F. Daviaud. Phys. Rev. Lett., 96, 204503 (2006). DOI: 10.1103/PhysRevLett.96.204503

[12] D. Zhilenko, O. Krivonosova, M. Gritsevich, P. Read. Chaos, 28, 053110 (2018). DOI: 10.1063/1.5011349

[13] M.L. Waite. Phys. Fluid., 29, 126602 (2017). DOI: $10.1063 / 1.5004986$

[14] U. Karban, B. Bugeat, E. Martini, F. Towne, A.V.G. Cavalieri, L. Lesshafft, A. Agarwal, P. Jordan, T. Colonius. J. Fluid Mech., 900, R5 (2020). DOI: 10.1017/jfm.2020.566

[15] M. Le Bars, D. Cebron, P. Le Gal. Annu. Rev. Fluid Mech., 47, 163 (2015). DOI: 10.1146/annurev-fluid-010814-014556

[16] M. Hoff, U. Harlander, C. Egbers. J. Fluid Mech., 789, 589 (2016). DOI: https://doi.org/10.1017/jfm.2015.743

[17] V.G. Kozlov, N.V. Kozlov, S.V. Subbotin. Acta Astr., 130, 43 (2017). DOI: http://dx.doi.org/10.1016/j.actaastro.2016.10.018

[18] Д.Ю. Жиленко, О.Э. Кривоносова. ЖТФ, 89 (7), 998 (2019). DOI: 10.21883/JTF.2019.07.47786.391-18 [D.Yu. Zhilenko, O.E. Krivonosova. Tech. Phys., 64 (7), 933 (2919).] DOI: $10.1134 / \mathrm{S} 106378421907032$

[19] Д.Ю. Жиленко, О.Э. Кривоносова. Письма в ЖТФ, 46 (12), 22 (2020). DOI: 10.21883/PJTF.2020.12.49522.18295

[D.Yu. Zhilenko, O.E. Krivonosova. Tech. Phys. Lett., 46 (12), 591 (2020).] DOI: 10.1134/S1063785020060292

[20] N. Nikitin. J. Comp. Phys., 217 (2), 759 (2006). DOI: $10.1016 /$ j.jcp.2006.01.036

[21] Д.Ю. Жиленко, О.Э. Кривоносова. Письма в ЖТФ, 39 (1), 62 (2013). [D.Yu. Zhilenko, O.E. Krivonosova. Tech. Phys. Lett., 39 (1), 84 (2013).] DOI: 10.1134/S1063785013010276

[22] Ю.Н. Беляев, И.М. Яворская. В сб. Течения вязкой жсидкости во вращающихся сферических слоях и их устойчивость, Итоги науки и техники. Сер. МЖГ, под ред. А.И. Михайлова (ВИНИТИ, М., 1980), т. 15, с. 3.

[23] R.R. Kerswell. J. Fluid Mech., 382, 283 (1999). DOI: https://doi.org/10.1017/S0022112098003954 
[24] Д.Ю. Жиленко, О.Э. Кривоносова. Письма в ЖЭТФ, 104, 552 (2016). DOI: 10.7868/S0370274X16200030 [D.Yu. Zhilenko, O.E. Krivonosova. JETP Lett., 104, 531 (2016). DOI: https://doi.org/ 10.1134/S0021364016200133]

[25] K. Nakabayashi, W. Sha, Y. Tsuchida. J. Fluid Mech., 534, 327 (2005). DOI: https://doi.org/10.1017/S0022112005004659

[26] Л.Д. Ландау, Е.М. Лившиц. Теоретическая физика. Т. 6. Гидродинамика. 3-е изд. (Наука, М., 1986)

[27] C. Lissandrello, L. Li, K.L. Ekinci, V. Yakhot. J. Fluid Mech., 778, R3 (2015). DOI: $10.1017 / \mathrm{jfm} .2015 .402$

[28] P.J. Schmid. Ann. Rev. Fluid Mech., 39, 129 (2007). DOI: 10.1146/annurev.fluid.38.050304.092139 